\title{
Foreword: prostate cancer in the elderly population
}

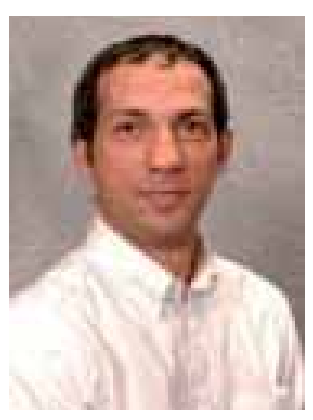

\section{Al B Barqami',} MD FRCS

Assistant Professor in U rology Director of Research Section of U rologic O ncology, U niversity of Colorado $\mathrm{H}$ ealth Science $C$ enter. Anschutz Cancer Pavillion 1665 N . U rsula Street, Suite 1004, P0 Box 6510, $M$ ail Stop F-710, Aurora, CO 80045, USA

Tel.: +1 7208480568 ;

Fax: +17208480203

E-mail: al.barqawi@ uchsc.edu
"O $\mathrm{f}$ all the self-fulfilling propheciesin our culture, the assumption that aging means dedine and poor health is probably the deadliett".

M arilyn Ferguson, The Aquarian Conspiracy, 1980

It is with great pleasure that I present this special edition of Aging $\mathrm{H}$ ealth, dedicated to prostate cancer in the elderly population.

Prostate cancer almost exclusively occurs in men aged 50 years and over. It has a tremendous impact on patients' quality of life, partially owing to its psychological burden but also, and more importantly, as a consequence of the treatment options currently available for its management. Prostate cancer is unique because of its potential to be 'outlived'. In other words, the majority of newly diagnosed prostate cancer cases will not be the cause of death, even without treatment. This is especially relevant in the elderly population owing to relatively shorter life expectancy and higher incidences of co-morbidities. H ence, the term 'clinically insignificant cancer' is commonly used to highlight this predicament in prostate cancer management.

'Prostate cancer is unique bec ause of its potential to be 'outlived'. In other words, the majority of newly diagnosed prostate cancercases will not be the cause of death, even without treatment.'

gen (PSA) screening, prevention strategies and the role of diet and $5 \alpha$-reductase inhibitors, accurate clinical staging and the efficient utilization of imaging techniques, to the best treatment options, which may include radical prostatectomy with or without lymph node dissection, various radiation therapy modalities and cryotherapy with or without hormonal blockade. $M$ any of these questions remain unanswered, partly owing to lack of strong clinical evidence, yet it is especially relevant to those individuals with advancing age because of the higher potential risk of overtreatment.
This special focus issue addresses and updates current concepts and clinical evidence on some of the controversial aspects surrounding prostate specific disease management relevant to the aging population. Screening in the elderly received special attention with a recently recommended publication from the US preventive services task force, questioning the validity of screening for prostate cancer using serum PSA levels in men over 75 years of age [1].

In this issue's editorial section, Dr M ouraview and $\mathrm{Dr}$ M oul discuss the evidence for and against age-based screening recommendations (pages 443-446). In more detail, Dr Wilson presents a comprehensive review on prostate cancer screening in the aging population. Dr Silbirstein and Dr Parsons are contributing to this issue by describing the current concepts and the role of diet in preventing and treating prostate cancer (pages 495-505). In addition, D r Yafi and his colleagues al so discuss the future prospects of chemoprevention of prostate cancer as well as current ongoing clinical studies in the USA and Europe (pages 469-479). It has long been the belief that hormonal manipulation may provide some survival benefit to prostate cancer survivors. H owever, recent literature emphasizes the significant side effects of castration and the questionable evidence for its widespread use, leading to new recommendations and limited utilization in a clinical based setting [2]. D rs Loeb and Schaeffer address the long standing controversy of the value of adjuvant hormonal blockade in men diagnosed with early stage disease.

In addition, $\mathrm{Dr}$ Art and $\mathrm{Dr}$ Holzbeierlein's broad review addresses in depth the evidence for hormonal and chemotherapeutic interventions in advanced stage prostate cancer (pages 455-462). Similarly, Dr Falci and his colleagues discuss the medical approach to advanced cancer, focusing on the 65 years and over age group (pages 481-494).

\section{Acknowledgements}

I wish to extend my sincere thanks to all the authors who contributed to this focus issue for their valuable time, effort and expertise in completing these reviews. I also wish to acknowledge the tremendous effort by the editorial staff; without them this task would not have been realized. It has been my privilege and honor to edit this focus issue. 
Financial \& competing interests disclosure The author has no relevant affiliations or financial involve ment with any organization or entity with a financial interest in or financial conflict with the subject matter or materials discussed in the manuscript. This includes employment, consultancies, honoraria, stock ownership or options, expert testimony, grants or patents received or pending, or royalties

$\mathrm{N}$ o writing assistance was utilized in the production of this manuscript.

\section{Bibliography}

1. Screening for prostate cancer: U S Preventive Services Task Force recommendation statement. Ann. Intern. M ed. 149(3), 185-191 (2008).

2. Lu-Yao GL, Albertsen PC, M oore D F: Survival following primary androgen deprivation therapy among men with localized prostate cancer. JAM A 300(2), 173-181 (2008). 